\title{
Health Information Systems and Registers
}

\author{
Boris A. Kobrinskii' ${ }^{1}$ Aleksey V. Breusov² \\ ${ }^{1}$ Federal Research Center “Computer Science and Control” of Russian Academy Science, Moscow, Russian Federation \\ ${ }^{2}$ Friendship University of Russia, Moscow, Russian Federation \\ Email: kba_05@mail.ru, ab69@yandex.ru
}

How to cite this paper: Kobrinskii, B.A. and Breusov, A.V. (2019) Health Information Systems and Registers. E-Health Telecommunication Systems and Networks, 8, 11-22.

https://doi.org/10.4236/etsn.2019.82002

Received: April 16, 2019

Accepted: June 10, 2019

Published: June 13, 2019

Copyright $\odot 2019$ by author(s) and Scientific Research Publishing Inc. This work is licensed under the Creative Commons Attribution International License (CC BY 4.0).

http://creativecommons.org/licenses/by/4.0/

\begin{abstract}
Health information systems are the basis of eHealth at regional and national levels. They provide the maintenance of traditional medical documents in outpatient clinics and hospitals in electronic form. At the same time, specialized registries are monitoring systems for the health status of people with certain diseases. However, the creation of registers as independent information systems, which takes place to this day, is now inadvisable, since the necessary information can be obtained from the Electronic Healthcare Record/ Electronic Medical Record. In this case, specialized registers are implemented as virtual systems of a temporary or permanent type. Regarding patient registers, they integrate data from electronic medical records and information from patients or their relatives.
\end{abstract}

\section{Keywords}

Modular Principle of Construction of Electronic Healthcare Records, Specialized Registers, Virtual Registers, eHealth

\section{Introduction}

The dilemma of the current situation is the independent implementation and functioning of national/regional health information systems (HIS), containing the data of all people living in a certain territory (geographically) and specialized registers for certain populations. This creates additional difficulties for an integrated analysis of patients' health status. In addition, the same person can be included in several registers (for example, if there is a congenital pathology in the child, information about it should be recorded in the register of congenital malformations and in the register of disabled persons). But in this case, the postulate of a single entry of data is violated and the burden on medical personnel is increased when data is entered. 
Recommendations on the principles of building large regional and national systems are developed without taking into account the requirements for specialized registers for various purposes. At the same time, the number of registers created in the world for various purposes is constantly increasing. They include rare diseases, treatment problems, mortality and many others [1] [2] [3] [4] [5]. Personal health and social data registers focused on the early detection of risk factors and the prevention of the bad for the health effects are also being increasingly used, including in Russia [6]. It is necessary to pay more attention to the exchange with registers of genetic information characteristic of certain diseases. The ClinGen Allele Registry addresses this problem by providing: 1) globally unique "canonical" variant identifiers (CAids); 2) access to variant-identifying information in a searchable registry; 3) links to allele-related records in many commonly used databases [7].

The purpose of this study was the problem of the formation of various registers on the basis of information accumulated during the life of an individual in the HIS. For this, it is proposed to use the modular principle of electronic medical records with the assignment of a permanent personal identifier at birth. At the same time, it is supposed to exchange with the personal medical records of people, the data in which come directly from patients or parents. This approach significantly reduces the time for register formation and provides more complete and high quality data in the registers. An important aspect can be considered the creation of national registries of rare diseases and their integration into higher level registers (European, American, Asian, international), which is especially important for the prevalence and diagnosis at a very low frequency of individual diseases and for the identification of new clinical forms of diseases. The novelty of the presented research lies in the automatic generation of various registers based on modules from Electronic Healthcare Record (EHR) data, which will speed up the quality of data exchange with HIS. In this case, specialized registers will be presented as virtual systems for solving problems in certain areas of medicine. If necessary, additional information, which is not available in standard EHRs, satellites medical or medical-social maps, including necessary information (for example, pedigrees and results of genetic studies, treatment methods in intensive care units, information on occupational hazards, etc.).

Thus, a fundamentally important difference in the formation of new HER/EMR and registers should be a modular principle of their construction. This will increase the speed of cross-border data exchange in the provision of medical care, including in emergency situations. Modules whose information does not matter at a certain age can be archived. In this case, the load on the search for the necessary information in the databases of regional and national systems will be reduced. At the same time, it will allow reforming and creating specialized registers based on the exchange of necessary modules, including not only with HIS, but also with personal registers, including those focused on the problem health optimization. This saves the time of physicians in the formation of a database of new registers. At the same time, the creation of registers for new 
areas will be possible with a significant reduction in time and labor costs for their development.

The subsequent presentation will provide an insight into the proposed structure of the interrelationships of electronic health records and registers, as well as the particularities and application of specialized registers in the modern eHealth system. The modular principle of building registers and automatically supported monitoring of the state of patients' health, data about which are contained in the databases of registers, are considered in detail.

Modular principle of construction of information medical systems

The unified concept of building national and regional information systems and specialized police registers for all levels of healthcare should imply the use of modular technology in eHealth [8] in a person-centred integration of the patient's lifetime medical data, which will be provided by patient-focused, patient-active and patient-confidential healthcare [9]. By a module we mean a standardized information structure that includes a fixed list of parameters. The module can contain attached text files and medical images. This approach involves the use of central ("metasystem") modules common to all health information systems (questionnaire, vital data, preventive vaccinations, transferable diseases, etc.) in combination with "intrasystem" modules for displaying a variety of information specific to various diseases and groups of the population (pregnant, neonatal period of life, occupational pathology, the course of certain diseases, etc.).

One of the most important "metasystem" modules, without which it is impossible to implement a person-centred approach and the construction of the Connected Healthcare Framework in the future, is the module of personal identifiers (PID) of the population, including the place of birth with names of parents. PID, formed in one way or another and assigned from birth, will ensure the subsequent integration of any medical information of the individual. The value of the module containing the unique personal identifier was shown in the UK's National Programme for IT [10]. This approach will allow the integration of all medical records and primary visual results of the research into a person, carried out in various organizations. At the same time, it will facilitate the collection of genealogical information necessary to determine the risk of hereditary predisposition to diseases.

A key component of eHealth is the Electronic Healthcare Record (EHR) or Healthcard [11], filled in from birth and updated by every medical organization that monitors, examines and treats a patient during his lifetime. With regard to in-system specialized modules that provide differentiation of information in the Electronic Healthcare Record/Electronic Medical Record (EMR), it is important to note the importance of their one-type structuring, which will allow rapid exchange of necessary data fragments on request. With the uniform principle of building EHR modules based on standards and classifiers of medical terms, confidence in the software product for medical workers will increase. At the same time, this will allow emergency medical teams and Disaster Medicine doctors to 
promptly access the necessary information stored in the integrated EHR in the format of individual modules.

The basic module of the EHR/EMR should contain information that is necessary for all physicians to access for examination and treatment in any situation. This information includes chronic diseases and certain acute diseases (myocardial infarction, hepatitis and others), vital characteristics and regular medications, as well as the results of fundamentally important studies. Satellite modules should be designed for displaying specific information about different conditions and diseases (pregnancy, occupational pathology, the course of certain chronic diseases, etc.) and particular treatments (chemotherapy, intensive treatment, etc.) as well as for storage of radiology images, etc. As certain information is age-dependent, personal medical data critical in early childhood loses its relevance after a certain point in time. The same happens with some of the information about acute diseases and injuries. Therefore, it seems expedient to create satellite EMRs in separate areas, some of which can be archived at a certain time, although this does not preclude their availability for viewing if necessary [8].

Satellite modules should also be provided for self-recording by citizens of information about their health and their children's health (development, nutrition, emotional state, stressful situations, physical training, etc.). In more detail, these issues will be discussed in the section on registers.

Basic and satellite EMR in conjunction with the Electronic Patient Record (EPR) or the Personal Healthcare Record/Patient Health Record (PHR) [12] is the basis for detecting minimal deviations in the health status of patients, for identifying primary manifestations of chronic diseases. The theoretical basis for this is the concept of the continuum of transitional states of the organism, suggesting an almost continuous process with no clear boundaries to the stages: the norm of the reaction - the functional deviations-the border states-the chronic diseases [13].

When creating a modular EMR structure, only necessary modules can be replicated from a central (or regional) server to local servers or the personal computers of medical organizations. This will allow any doctor to request and receive exactly the information he needs at the moment, in his field, brought together on the basis of observation of the patient at different times in different medical organizations for a certain pathology (for example, a cardiologist has no need for detailed information in the field of urology). At the same time, all information about the transferred and existing chronic diseases can be viewed (received) from a single module "Diagnoses".

The modular principle of EMR construction will allow, if necessary, the formation of regional and national information medical subsystems on the basis of only those modules that are necessary for monitoring the state of health of a certain contingency (for example, in environmental or man-made disasters), generating various reports and making managerial decisions. Modules containing current clinical manifestations of diseases will be stored in HIS databases or 
regional Cloud Optimized Storage and Computing.

This approach will ensure:

- Authorized access by medical workers to the necessary EMR/EHR modules and medical images (MRI, ultrasound, etc.) in accordance with their functions, on the basis of the role model of access control, including on-line use for obtaining a second opinion;

- The opportunity for citizens to have modules for personal monitoring of their health status and fixing self-directed health-improving/corrective measures (with authorized access by the attending physicians to the patient's personal modules).

\section{Registers in eHealth}

Clinical registries are recognized as a key element of health informatization [14]; they are the most accurate and convenient method for monitoring the quality of care and benchmarking. Rare disease registries are a priority at European level and specific actions are being implemented by the European Commission to support their development. For example, in Italy, a National Registry of rare diseases has been established as a network of regional registries [15]. The Joint Declaration EURORDIS-NORD-CORD formulates ten Key Principles of Rare Disease Patient Registries [16]. Among these principles, in the context of this article, the following recommendations consider: 1) covering, if possible, broad geographic regions, register interaction, 2) using a set of common uniform elements, 3) including data from patients, along with those received from doctors. It should be noted that, for example, the national American data system for burns includes information on emergency care for burned patients were used to establish standards of care for burned patients and to indicate the methods used by more successful hospitals [17]. A similar principle of integration and data exchange forms the register of acute coronary syndromes of the European Cardiological Society [18]. In a study conducted in the United States examines in detail the various aspects of the design and use of registers, focusing on the study of the efficacy and safety of medicines in their long-term use in wide-ranging trials [19]. Researchers pay attention to the analysis of the appropriateness and safety of medications, based on the information accumulated in the registers on their use.

Figure 1 shows a register circuit that can be formed from EMR modules and data input by individuals. Users of such a register are both doctors and patients. In specific cases, certain modules may be omitted from the register.

On this basis, a single global register network for different diseases can be created, which was barely realized in practice when creating autonomously functioning problem-oriented registers formed independently of EMR. Our proposed solution allows us to combine any data, similar to the Treat-NMD register system launched in early 2007 for neuromuscular diseases [20]. As noted by the author, this may allow us to understand similar processes and their correlation with the results of treatment of patients. 


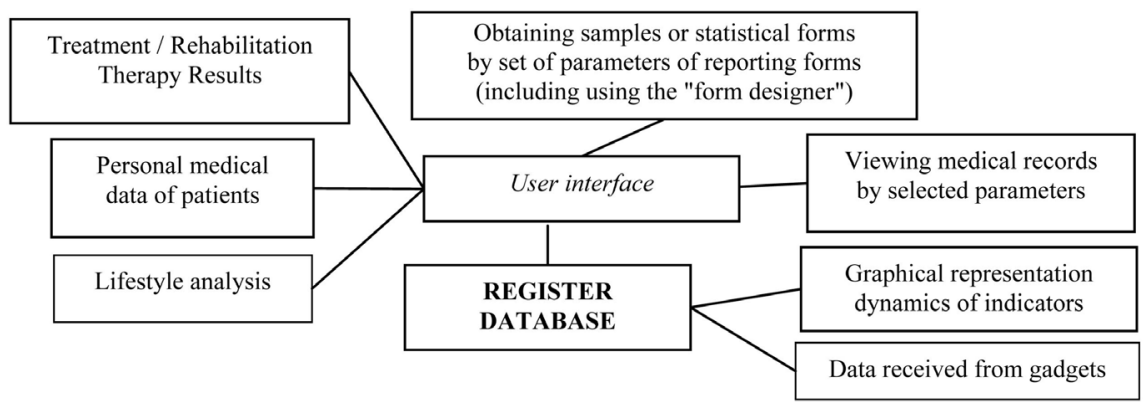

Figure 1. Typical scheme of a modular register.

Systems that monitor the effectiveness of treatment involves applying them both to individual medical organizations and integrating data useful to a community of doctors using similar therapies. It is also necessary for research purposes in relation to rare diseases that are impossible or difficult to treat within the same institution. This, for example, was noted by the so-called global register of patients with type II mucopolysaccharidosis [21]. The authors note the importance of monitoring the safety and efficacy of enzyme therapy, with the possibility of referring physicians to a central database that provides access to information from a large number of patients with mucopolysaccharidosis-II to better understand the long-term effects of specific therapy. The use of unified medical records, the data of which will be copied into a single register, is a condition for maintaining a high degree of continuity in pathogenetic therapy. In Sweden, two mutually complementary spine pathology registries are used to compare the efficacy of treating degenerative vertebral disorders [22]. According to the authors, the further development of the system for collecting data on patients with this pathology will make it possible to better illuminate treatment in various clinical variants encountered in patients with these complex diseases.ith these complex diseases.

\section{Formation of Specialized Registers in the Framework of the Modular Concept of Building Information Systems}

The formation of specialized registers in the form of modular integration can be provided by sending copies of separate fragments of EMR from the regional Cloud Optimized Storage and Computing to the national data centre. The postulated approach should provide for a transition to multilateral monitoring of individual health of people and dynamic analysis of the demographic situation (population monitoring) based on full information coming from health organizations at all levels.

Personal (police) registers for certain contingencies are needed not so much for statistical analysis, which can be carried out by forming the necessary samples in Cloud Optimized Storage and Computing at any level, but for monitoring families with hereditary and congenital diseases (genetic registers), persons injured in technogenic catastrophes (ecopathological registers), for monitoring patients at risk of socially significant pathology and being on certain treatment 
(oncological, cardiological, HIV/AIDS, etc. registers). The modular principle of HIS construction will make it possible to overcome the present "conflict" between horizontal (regional) systems and vertical (national) specialized registers by type of pathology or social group.

In the technology of the modular architecture of electronic medical records, registers can be considered as functional or conditionally virtual specialized HIS. Their "assembly" will be performed in Cloud Optimized Storage and Computing at the appropriate level, based on the EMR and PHR data records for the current or selected time point (Figure 2). For newly created registers, it will be necessary to sample the necessary data from different EMR individuals with this pathology and/or, at the very least, create an additional satellite module EMR.

Integration of the genealogical information modules is especially important. With the elements of pedigrees, EHR of individual family members should be linked. This will allow us to timely assess the risk of hereditary (monogenic, chromosomal) diseases and multifactorial diseases, and plan personalized preventive measures. In the long term it will allow uniting in uniform pedigrees information about the health of relatives living in different regions and countries.

The new principle of the formation of specialized registers will allow fast resolution of the tasks of the medical and social plans for healthcare. An example of a system that works in this direction is the monitoring of immunization through the US regional and state registries by unidirectional links of immunization data from the EMR to the relevant registries [23]. Simultaneously, on this basis, it will be possible to carry out multicentre studies on large regional and national data sets (according to the type of the international register of deep brain stimulation in Parkinson's disease [24]).

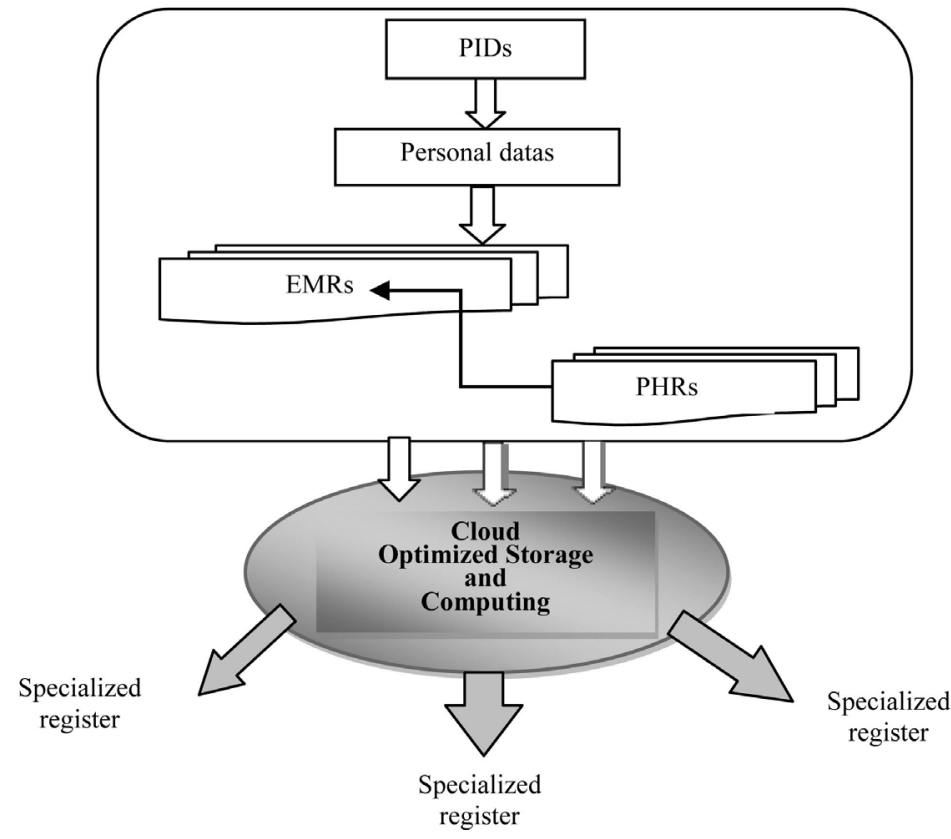

Figure 2. The movement of information in the system "EMR/HER-PHR-Cloud Optimized Storage and Computing-Specialized personalized medical registers". 


\section{Results}

The modular principle of building medical information systems is the basis for speeding up the creation of new or correction of existing registers. A significant part of the register system blocks in this case is already available in the HIS. Virtual registers, provided they are generated using HIS modules, will be created and modified automatically in accordance with the proposed algorithms. This will prevent re-entry of data into registers. In quantitative terms, based on Russian data, this approach will reduce the amount of duplicate information input by $60 \%-75 \%$. These results are based on the analysis of information that is entered into the Russian registers of congenital malformations, children with disabilities and children with mucopolysaccharidoses of the first and second types receiving enzyme-substituting drugs.

\section{Discussion}

Many registers do not store data on the course of the pathological process. In this respect the opinion of M.I. Bellgard et al. [25], is that the bottleneck of registers is static, while continuous analysis of the data could help improve the health of patients. The uniformity and structure of clinical information is also required. This corresponds to the first step in using common tools for collecting comparable data on the type of a single set of elements for a European registration platform for patients with rare diseases [26]. The proposed system of uniform entering of information about patients promotes more complete information, with the possible participation of patients and patient associations in the process of data registration. The same objective is served by the decision of the French Reference Centres for Orphan Diseases to unite under a single information system called CEMARA [27]. According to American experts, full-scale observational data for cystic fibrosis, which can be regularly obtained from HER, are observational data is important for clinical research, quality improvement, and clinical management [1]. Similarly, the international public database and the Tourette International Syndrome Registry, when obtaining additional information from electronic medical records, will help increase the efficiency and safety of deep brain stimulation in this disease [3].

At the European level [28] proposed using the basic minimum list of elements, which should become an obligatory data set for all European registers at the national level. Other data necessary for the health of each country for their specific purposes can be added in national registers. This approach is analogous to the modular principle of the formation of registers of various types discussed above, based on the extraction of unified standardized data from patient-oriented EHR and other data.

The role of patients in taking care of their own health is being given increasing importance at present, various aspects of patient participation in the active prevention of the development of diseases are being considered [29]. Personal systems for assessing the risk of disease and preventing their development are also 
complemented by data from the EHR modules. This approach is also applied in Russia [30]. Thus, a modular approach to the construction of HIS and various specialized registers is becoming more widely used in medical practice.

The problem of the death of young people (SDY) was the cause of creating a special register. Information can come from various sources, in particular, from electronic medical records. This will help identify risk factors for sudden death in the young. (SDY register objectives are to: 1) describe the incidence of SDY in the United States by using population-based surveillance; 2) compile data from SDY cases to create a resource of information and DNA samples for research;3) encourage standardized approaches to investigation, autopsy, and categorization of SDY cases. The SDY case registry will provide valuable incidence data and will enhance understanding of the characteristics of SDY cases to inform the development of targeted prevention efforts as part of health preservation system [31].

\section{Conclusions}

On the basis of the unified concept of the modular construction of the EHR/EMR, a multi-level pyramid of the information space of a public health protection system can be created, where they can contribute information about the state of health from citizens or parents of children and children from a certain age. This will allow integrating the necessary modules to form permanently functioning or virtual specialized registers using any information previously entered into the EMR.

A new look at the formation of registers should include the integration of information from EHR, PHR and data of mHealth [32]. This will provide regular, objective information about the health of individuals, to monitor theirs by creating specialized medical registers.

\section{Conflicts of Interest}

The authors declare no conflicts of interest regarding the publication of this paper.

\section{References}

[1] Knapp, E.A., Fink, A.K., Goss, C.H., Sewall, A., Ostrenga, J., Dowd, C., Elbert, A., Petren, K.M. and Marshall, B.C. (2016) The Cystic Fibrosis Foundation Patient Registry. Design and Methods of a National Observational Disease Registry. Annals of the American Thoracic Society, 13, 1173-1179.

https://www.ncbi.nlm.nih.gov/pubmed/27078236 https://doi.org/10.1513/AnnalsATS.201511-7810C

[2] Aksamit, T.R., O’Donnell, A.E., Barker, A., Olivier, K.N., Winthrop, K.L., Daniels, L.A., Johnson, M., Eden, E., Griffith D., Knowles, M., Metersky, M., Salathe, M., Thomashow, B., Tino, G., Turino, G., Carretta, B. and Daley, C.L. (2017) Adult Patients with Bronchiectasis: A First Look at the US Bronchiectasis Research Registry. Chest, 151, 982-992. https://www.ncbi.nlm.nih.gov/pubmed/27889361 https://doi.org/10.1016/j.chest.2016.10.055

[3] Martinez-Ramirez, D., Jimenez-Shahed, J., Leckman, J.F., Porta, M., Servello, D., 
Meng, F.-G., Kuhn, J., Huys, D., Baldermann, J.C., Foltynie, T., Hariz, M.I., Zrinzo, L., Kefalopoulou, Z., Silburn, P., Coyne, T., Mogilner, A.Y., Pourfar, M.H., Khandhar, S.M., Auyeung, M., Ostrem, J.L., Visser-Vandewalle, V., Welter, M.-L., Mallet, L., Karachi, C., Houeto, J.L., Klassen, B.T., Temel, Y., Gross, R.E., Walker, H.C., Lozano, A.M., Walter, B.L., Mari, Z., Anderson, W.S., Changizi, B.K., Moro, E., Zauber, S.E., Schrock, L.E., Zhang, J.-G., Hu, W., Rizer, K., Monari, E.H., Foote, K.D., Malaty, I.A., Deeb, W., Gunduz, A. and Okun, M.S. (2018) Efficacy and Safety of Deep Brain Stimulation in Tourette Syndrome. The International Tourette Syndrome Deep Brain Stimulation Public Database and Registry. Journal American Medical Association Neurology, 75, 353-359.

https://www.ncbi.nlm.nih.gov/pubmed/29340590

https://doi.org/10.1001/jamaneurol.2017.4317

[4] Leslie, W.D., Johansson, H., McCloskey, E.V., Harvey, N.C., Kanis, J.A. and Hans, D. (2018) Comparison of Methods for Improving Fracture Risk Assessment in Diabetes: The Manitoba BMD Registry. Journal of Bone and Mineral Research, 33, 1923-1930. https://www.ncbi.nlm.nih.gov/pubmed/29953670 https://doi.org/10.1002/jbmr.3538

[5] Lopez, K.N., Nembhard, W.N., Wang, Y., Liu, G., Kucik, J.E., Copeland, G., Gilboa, S.M., Kirby, R.S. and Canfield, M. (2018) Birth Defect Survival for Hispanic Subgroups. Birth Defects Research, 110, 352-363. https://doi.org/10.1002/bdr2.1157 https://www.ncbi.nlm.nih.gov/pubmed/29195034

[6] Grigoriev, O.G. and Molodcenkov, A.I. (2018) Risk Health Evaluation and Selection of Preventive Measures Plan with the Help of Argumental Algorithm. In: Kuznetsov, S., Osipov, G.S. and Stefanuk, V., Eds., Artificial Intelligence. RCAI 2018. Communications in Computer and Information Science, Springer, Cham, 280-290. https://www.springer.com/gp/book/9783030006167 https://doi.org/10.1007/978-3-030-00617-4_26

[7] Pawliczek, P., Patel, R.Y., Ashmore, L.R., Jackson, A.R., Bizon, C., Nelson, T., Powell, B., Freimuth, R.R., Strande, N., Shah, N., Paithankar S., Wright, M.W., Dwight, S., Zhen, J., Landrum, M., McGarvey, P., Babb, L., Plon, S.E. and Milosavljevic, A. (2018) ClinGen Allele Registry Links Information about Genetic Variants. Human Mutation, 39, 1690-1701. https://www.ncbi.nlm.nih.gov/pubmed/30311374 https://doi.org/10.1002/humu.23637

[8] Kobrinskii, B.A. (2014) E-Health and Telemedicine: Current State and Future Steps. E-Health Telecommunication Systems and Networks, 3, 50-56.

https://file.scirp.org/pdf/ETSN 2014120511312331.pdf https://doi.org/10.4236/etsn.2014.34007

[9] Dawson, J., Tulu, B. and Horan, T.A. (2009) Towards Patient-Centered Care: The Role of E-Health in Enabling Patient Access to Health Information. In: Wilson, E.V., Ed., Patient-Centered E-Health, Information Science Reference Hershey, New York, 1-9. https://www.danangtimes.vn/Portals/0/Docs/112144754-1605660167.pdf https://doi.org/10.4018/978-1-60566-016-5.ch001

[10] Swindells, M. and De Lusignan, S. (2012) Lessons from the English National Programme for IT about Structure, Process and Utility. Studies in Health Technology and Informatics, 174, 17-22.

https://www.iospress.nl/book/large-scale-projects-in-ehealth/

[11] Brooks, P. and Health, A. (2010) Standards and Interoperability in Healthcare Information Systems: Current Status, Problems, and Research Issues. Proceedings of the 5 th Midwest Association for Information Systems Conference, Moorhead, MN, 21-22 May 2010, 18. http://aisel.aisnet.org/mwais2010/18 
[12] Wolf, S.M. and Evans, B.J. (2018) Return of Results and Data to Study Participants. Science, 362, 159-160. https://www.ncbi.nlm.nih.gov/pubmed/30309935 https://doi.org/10.1126/science.aav0005

[13] Kobrinsky, B. (1995) Concept of the Continuum of Intermediate States of Development: Risk Factors in Child Health. Medical Audit News, 2, 21-22.

[14] Bufalino, V.J., Masoudi, F.A., Stranne, S., Bufalino, V.J., Masoudi, F.A., Stranne, S.K., Horton, K., Albert, N.M., Beam, C., Bonow, R.O., Davenport, R.L., Girgus, M., Fonarow, G.C., Krumholz, H.M., Legnini, M.W., Lewis, W.R., Nichol, G., Peterson, E.D., Rumsfeld, J.S., Schwamm, L.H., Shahian, D.M., Spertus, J.A., Woodard, P.K. and Yancy, C.W. (2011) The American Heart Association's Recommendations for Expanding the Applications of Existing and Future Clinical Registries: A Policy Statement from the American Heart Association. Circulation, 123, 2167-2179.

https://www.ncbi.nlm.nih.gov/pubmed/21482960

https://doi.org/10.1161/CIR.0b013e3182181529

[15] Taruscio, D., Kodra, Y., Ferrari, G. and Vittozzi, L. (2014) The Italian National Rare Diseases Registry. Blood Transfusion, 12, s606-s613. https://www.ncbi.nlm.nih.gov/pubmed/24922301

[16] EURORDIS-NORD-CORD (2012) Joint Declaration 10 Key Principles of Rare Disease Patient Registries.

http://download.eurordis.org/documents/pdf/EURORDIS NORD CORD JointDe c Registries FINAL.pdf

[17] Feller, I. and Jones, C.A. (1987) The National Burn Information Exchange. The Use of a National Burn Registry to Evaluate and Address the Burn Problem. Surgical Clinics of North America, 67, 167-189.

https://www.sciencedirect.com/science/article/pii/S003961091644140X https://doi.org/10.1016/S0039-6109(16)44140-X

[18] Bradshaw, P.J., Ko, D.T., Newman, A.M., Donovan, L.R. and Tu, J.V. (2006) Validity of the GRACE (Global Registry of Acute Coronary Events) Acute Coronary Syndrome Prediction Model for Six Month Post-Discharge Death in an Independent Data Set. Heart, 92, 905-909. https://www.ncbi.nlm.nih.gov/pubmed/16387810 https://doi.org/10.1136/hrt.2005.073122

[19] Gliklich. R., Dreyer, N. and Leavy, M. (2014) Registries for Evaluating Patient Outcomes: A User's Guide. 3rd Edition, Agency for Healthcare Research and Quality Publication, Rockville, MD, 295.

https://effectivehealthcare.ahrq.gov/sites/default/files/related files/registries-guide3rd-edition-vol-2-140430.pdf

[20] Bushby, K., Lynn, S. and Straub, V. (2009) Collaborating to Bring New Therapies to the Patient-The TREAT-NMD Model. Acta Myologica, 28, 12-15. https://www.ncbi.nlm.nih.gov/pmc/articles/PMC2859629/

[21] Wraith, J.E., Beck, M., Giugliani, R., Clarke, J., Martin, R. and Muenzer, J. (2008) Initial Report from the Hunter Outcome Survey. Genetics in Medicine, 10, 508-516. https://www.ncbi.nlm.nih.gov/pubmed/18580692 https://doi.org/10.1097/GIM.0b013e31817701e6

[22] Lake, W.B., Brooks, N.P. and Resnick, D.K. (2013) Comparative Effectiveness Research in Spine Surgery. Journal of Comparative Effectiveness Research, 2, 45-51. https://www.ncbi.nlm.nih.gov/pubmed/24236520 https://doi.org/10.2217/cer.12.73

[23] Stevens, L.A., Palma, J.P., Pandher, K.K. and Longhurst, C.A. (2013) Immunization Registries in the EMR Era. Online Journal of Public Health Informatics, 5, 211. https://www.ncbi.nlm.nih.gov/pubmed/23923096 
https://doi.org/10.5210/ojphi.v5i2.4696

[24] Deuschl, G., Jain, R., Wang, A. and Scholtes, H. (2019) Outcomes of a Prospective, Multicenter, International Registry of Deep Brain Stimulation for Parkinson's Disease. Neurology, 92, 15.

https://n.neurology.org/content/92/15 Supplement/P1.8-039

[25] Bellgard, M.I., Render, L., Radochonski, M. and Hunter, A. (2014) Second Generation Registry Framework. Source Code for Biology and Medicine, 9, 14-19.

https://scfbm.biomedcentral.com/articles/10.1186/1751-0473-9-14 https://doi.org/10.1186/1751-0473-9-14

[26] Taruscio, D., Mollo, E., Gainotti, S., De la Paz, M.P., Bianchi, F. and Vittozzi, L. (2014) The EPIRARE Proposal of a Set of Indicators and Common Data Elements for the European Platform for Rare Disease Registration. Archives of Public Health, 72, 35-42. https://www.ncbi.nlm.nih.gov/pmc/articles/PMC4210567/ https://doi.org/10.1186/2049-3258-72-35

[27] Messiaen, C., Le Mignot, L., Rath, A., Richard, J.B., Dufour, E., Ben Said, M., Jais, J.P., Verloes, A., Le Merrer, M., Bodemer, C., Baujat, G., Gerard-Blanluet, M., Bourdon-Lanoy, E., Salomon, R., Ayme, S. and Landais, P. (2008) CEMARA: A Web Dynamic Application within a N-Tier Architecture for Rare Diseases. Studies in Health Technology and Informatics, 136, 51-56.

https://www.ncbi.nlm.nih.gov/pubmed/18487707

https://doi.org/10.1016/j.respe.2008.02.096

[28] EUCERD (2015) Minimum Data Set for Rare Disease Registries. EUCERD Joint Action WP8 TASK 8.1: Integration of Registry Activities. http://www.eucerd.eu/wp-content/uploads/2015/03/WP8 Registries MDS.pdf

[29] Gliklich, R.E., Dreyer, N.A, Leavy, M.B. and Christian. J.B. (2018) Registries for Evaluating Patient Outcomes: A User's Guide: 3rd Edition, Addendum. Agency for Healthcare Research and Quality, Rockville, MD, 103.

https://www.ncbi.nlm.nih.gov/pubmed/29708678

[30] Grigoriev, O.G., Kobrinskii, B.A., Osipov, G.S., Molodchenkov, A.I. and Smirnov, I.V. (2018) Health Management System Knowledge Base for Formation and Support of a Preventive Measures Plan. Procedia Computer Science, 145, 238-241.

https://doi.org/10.1016/j.procs.2018.11.050 https://www.sciencedirect.com/science/article/pii/S1877050918323366

[31] Burns, K.M., Bienemann, L., Camperlengo, L., Cottengim, C., Covington, T.M., Dykstra, H., Faulkner, M., Kobau, R., Lambert, A.B., MacLeod, H., Parks, S.E., Rosenberg, E., Russell, M.W., Shapiro-Mendoza, C.K., Shaw, E., Tian, N., Whittemore, V. and Kaltman, J.R. (2017) The Sudden Death in the Young Case Registry: Collaborating to Understand and Reduce Mortality. Pediatrics, 139, e20162757. https://www.ncbi.nlm.nih.gov/pubmed/28228502 https://doi.org/10.1542/peds.2016-2757

[32] Kelli, H.M., Witbrodt, B. and Shah, A. (2017) The Future of Mobile Health Applications and Devices in Cardiovascular Health. European Medical Journal Innovations, 1, 92-97. https://www.ncbi.nlm.nih.gov/pubmed/28191545 